\title{
Latent Finger Print Matching using FFT and DCT
}

\author{
P. Sony \\ Assistant Professor, Dept of \\ ECE \\ Vishnu Institute of Technology \\ A.P, India
}

\author{
K. Kiran \\ Assistant professor, Dept of \\ ECE \\ Vishnu Institute of Technology \\ A.P, India
}

\author{
R. Bharat Kumar \\ Assistant professor, Dept of \\ ECE \\ Vishnu Institute of Technology \\ A.P,India
}

\begin{abstract}
Human fingerprints be affluent in particulars called minutiae, which be able to used as credentials marks for fingerprint authentication. The objective of this project is to build up a complete system for fingerprint verification through extracting and matching minutiae. To achieve excellent minutiae extraction in fingerprints with varying quality, pre-processing in form of image enhancement and binarization is first applied on fingerprints before they are evaluated. Several methods have been joint to build a minutia extractor and a minutia matcher. Minutia-marking with false minutiae removal methods are used in the work. An alignment based elastic matching algorithm has been urbanized for minutia matching. This algorithm is capable of finding the correspondences between input minutiae pattern and the stored template minutia pattern without resorting to comprehensive search. Performance of the developed system is carried out by using FFT and DCT, and the results are analyzed on a data base with fingerprints from different people
\end{abstract}

\section{Keywords}

Image Enhancement, Minutiae Extraction, False minutiae, Minutiae matching, FFT, DCT.

\section{INTRODUCTION}

A fingerprint is an impression left by the friction ridges of primate hand or any part of a human. The foot print can also leave the friction ridges. The raised part of the epidermis lying on the digits (toes $\&$ fingers) is known friction ridges, the sole of the foot otherwise the palm of the hand, consisting of number of ridges joined as friction ridge skin. These ridges are also called as "ridges of epidermal ".

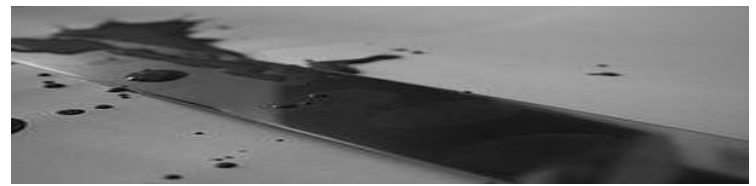

Figure 1.1: Barely visible Latent prints on a knife

Latent fingerprints obtained as of offense scenes have served as essential indication in forensic detection for more than a century. However, there have been instances where mistakes in latent fingerprint identification have led to criminal convictions.

One of the most high profile cases in which such a mistake was made in the case of Brandon Mayfield who was wrongly apprehended in the Madrid train bombing incident after a latent fingerprint obtained from the bombing site was incorrectly matched with his fingerprint in the FBI database.

The manual latent identification process can be divided into four steps, namely, (1) analysis, (2) comparison, (3) evaluation, and (4) verification.

Analysis refers to assessing the latent fingerprint to determine whether sufficient ridge information is present in the image to be processed and to mark the features along with the associated quality information. The latent print analysis is usually performed manually by a human expert. Similarity refers to a stage where an examiner compares a latent image to a reference print to determine their similarities or dissimilarities. Fingerprint features at every single one levels are compared at this stage.

Evaluation stage refers to classifying the fingerprint pair as individualization (identification or match), exclusion (no match) or in exclusive.

Verification is the process of reexamination of a fingerprint pair independently by another examiner in order to verify the results of the first examiner.
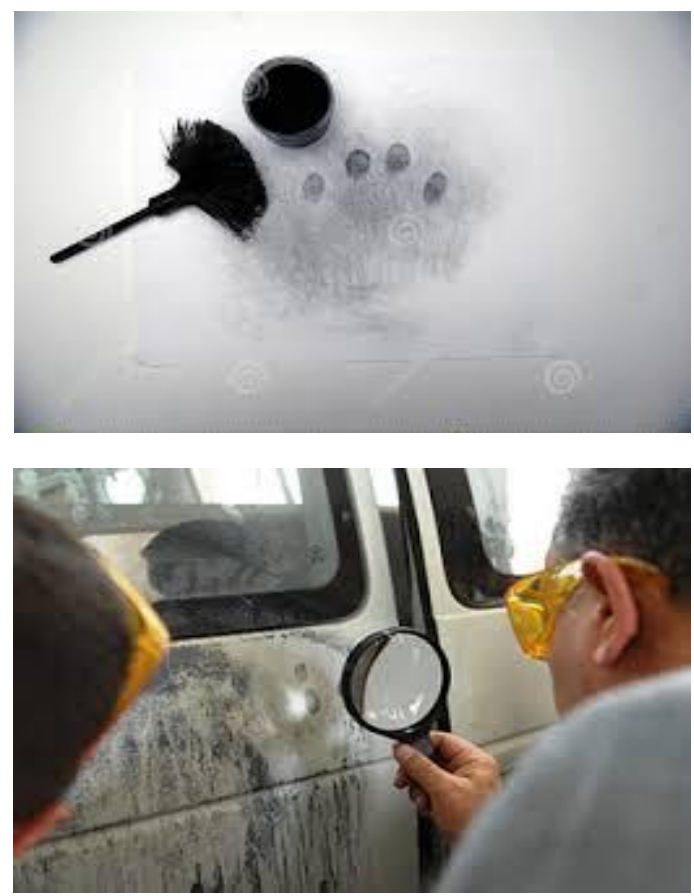

Figure 1.2: Collection of latent from ground \&car

\section{PROPOSED WORK}

A comprehensive minutiae extraction design for involuntary fingerprint recognition systems is presented. The proposed technique uses humanizing alternatives for the image enhancement process, leading accordingly to an increase of the reliability in the minutiae extraction task. 


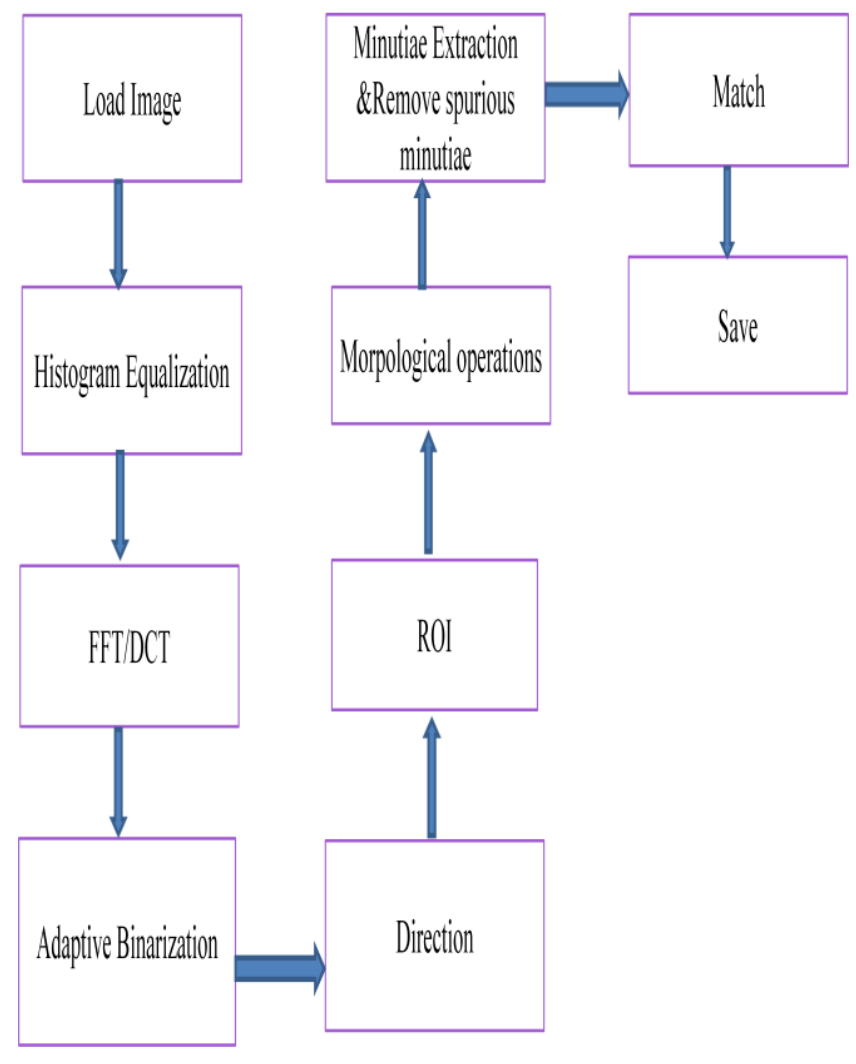

Figure 2.1: Steps involved in Latent finger print matching

In the third step FFT/DCT either of the algorithm is used at a time. We analyzed results with FFT and DCT. In the step seventh Morphological operations refers to Thinning, Removal of spikes and removal of $\mathrm{H}$ breaks.

Two Methods are adopted for image enhancement stage:

The first one is Histogram Equalization the next one is FFT/DCT.

\section{A. Fingerprint Image Enhancement}

Fingerprint Image enhancement is to make the image clearer for easy further operations. Since the fingerprint images acquired from sensors or other medias are not assured with perfect quality, those enhancement methods, for increasing the contrast between ridges and for rows and for connecting the false broken points of ridges due to insufficient amount of ink, are very useful for keep a higher accuracy to fingerprint recognition.

\section{Histogram Equalization}

Histogram equalization is to expand the pixel value distribution of an image so as to increase the perception information. The original histogram of a fingerprint image has the bimodal type,the histogram after the histogram equalization occupies all the range from 0 to 255 and the visualization effect is enhanced.

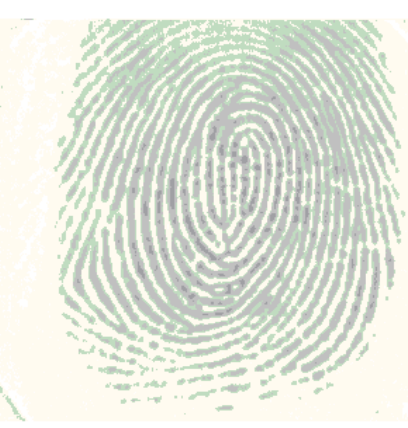

Figure 2.2: Original Image

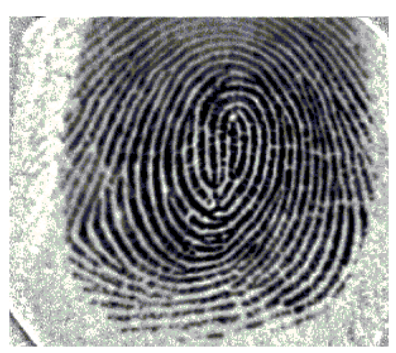

Figure 2.3: Image after Histogram Equalization

\section{IMAGE TRANSFORMS}

A. Fast Fourier Transform (FFT)

In the image enhancement two operations are performed they are histogram equalization and FFT as shown in equation 4.10. The fingerprints having $b$ the ridges to both white ridges and black ridges the information available in both the ridges to receive the information histogram equalization is used.

The Fourier transform is applied for the pixel based on divide the image into small processing blocks( 32 by 32 pixels).

$$
\begin{gathered}
F(U, V)=\sum_{x=0}^{M-1} \sum_{y=0}^{N-1}{ }_{y=0}^{f(x, y)} \exp \left\{-j 2 \pi^{*}[u x / M+v y / N)\right\}----(3.1) \\
\text { For } \mathrm{u}=(0,1,2, \ldots, 31) \text { and } \mathrm{v}=(0,1,2, \ldots, 31) .
\end{gathered}
$$

By the dominant frequencies enhancement the specific block the magnitude is multiply with the FFT of the block a set of times

Magnitude of the original is

$$
F(U, V)=a b s(F(u, v))=|F(u, v)| \text {. }
$$

We get the enhanced block according to $g(x, y)=F^{l}\left\{F(u, v) *|F(u, v)|^{k}\right.$.

Where $\mathrm{F}^{-1}(\mathrm{~F}(\mathrm{u}, \mathrm{v}))$ is done by:

$f(x, y)=1 / M N \sum_{x=0}{ }^{M-l} \sum_{y=0}{ }^{N-1} F(u, v) * \exp \left\{j 2 \pi^{*}[u x / M+v y / N]\right\}---(3.4)$ for $\mathrm{x}=(0,1,2, \ldots, 31)$ and $\mathrm{y}=(0,1,2, \ldots, 31)$.

Thus a termination might become a bifurcation Figure 3.1(a),(b) represents the original image and the image after FFT enhancement respectively 


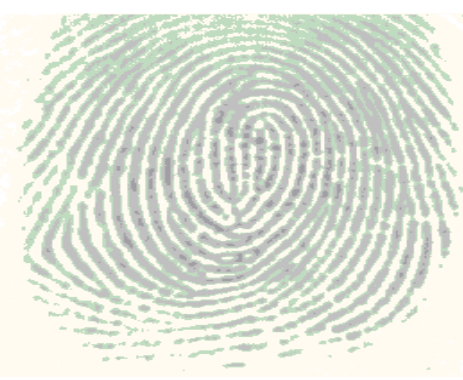

Figure 3.1: Original image

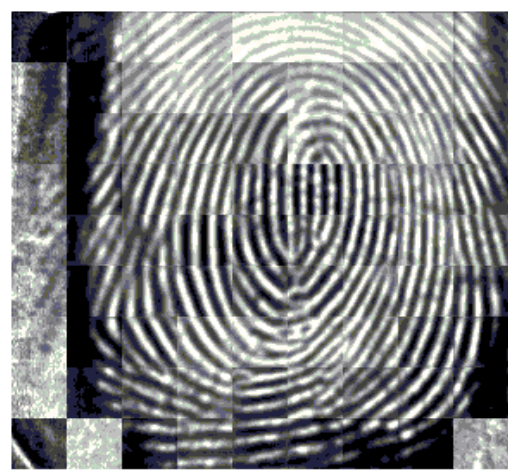

Figure 3.2: Fingerprint after FFT

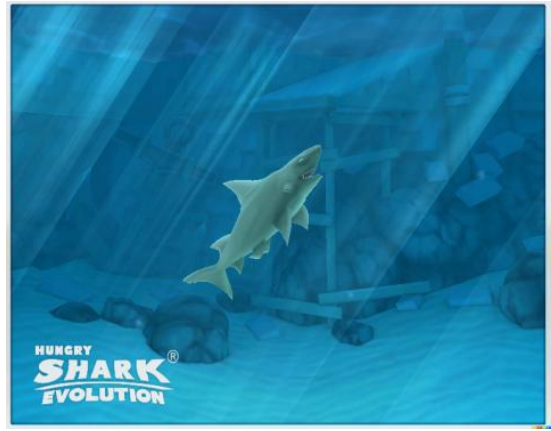

Figure 3.3: Original image

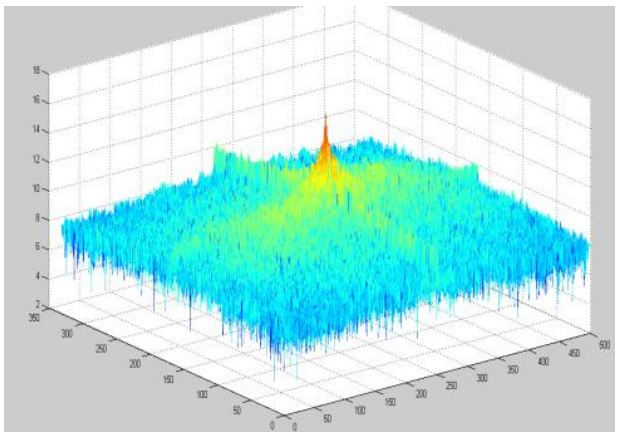

Figure 3.4: 3D plot of FFT

\section{B. DISCRETE COSINE TRANSFORM (DCT)}

The Discrete Cosine Transform was introduced by Ahmed et al in 1974, is one of the extensive family of sinusoidal transform. The concept of this transformation is to transform as set of points from the spatial domain into an identical representation in a frequency domain. The two dimensional DCT as shown in equation $(3.5 \& 3.6])$ then can be written in terms of pixel values $f(i, j)$ for $i, j=0,1 \ldots \ldots . . . N-1$ and the frequency-domain transform coefficients $\mathrm{F}(\mathrm{u}, \mathrm{v})$.
$\mathrm{F}(\mathrm{u}, \mathrm{v})=$

$$
\begin{aligned}
& \frac{1}{\sqrt{2 N}} c(u) c(v) \sum_{i=0}^{N-1} \quad \sum_{j=0}^{N-1} f(i, j) x \cos \left[\frac{(2 i+1) u \pi}{2 N}\right] \cdot \cos \left[\frac{(2 j+1) v \pi}{2 N}\right] \\
& - \text { Where } \mathrm{C}(\mathrm{u}), \mathrm{C}(\mathrm{v})=\{1 / \sqrt{ } 2\} \text { For u,v }=0 \\
& \mathrm{C}(\mathrm{u}), \mathrm{C}(\mathrm{v})=1 \quad \text { Otherwise } \\
& \mathrm{f}(\mathrm{i}, \mathrm{j})= \\
& \sum_{u=0}^{N-1} \quad \sum_{v=0}^{N-1} c(u) c(v) F(u, v) x \cos \left[\frac{(2 i+1) u \pi}{2 N}\right] \cdot \cos \left[\frac{(2 i+1) v \pi}{2 N}\right]
\end{aligned}
$$$$
\text { for } \mathrm{i}, \mathrm{j}=0,1 \ldots . . \mathrm{N}-1
$$

$f(i, j)$ is the $i, j^{\text {th }}$ element of the image (intensity of the pixel) represented by the matrix $f$.

$\mathrm{N}$ is the size of block DCT is done on here $\mathrm{N}=8$. Frequency coordinates in the transform domain are u, v. As Figure 8(b) each sub block contains one DC coefficient and other AC coefficient that shows lower frequency coefficients that contain (useful data for the image) gather at top-left corner and high frequency ones that contain less useful information at the bottom-right corner of the transformed matrix.

\section{Difference between DFT and DCT}

When using DFT, programmers should perform complex computation however by using DCT. This complexity in computations can be reduced by usage of real numbers.

Figure 6 (a), (b) represents the original image and the image after DCT enhancement respectively

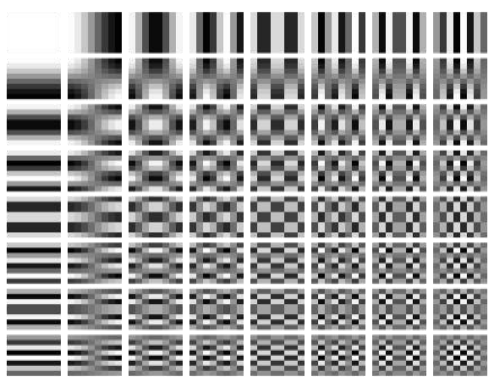

Figure 3.5: Two Dimensional DCT frequencies

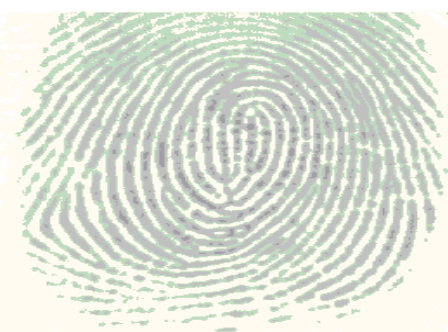

Figure 3.6: Original image

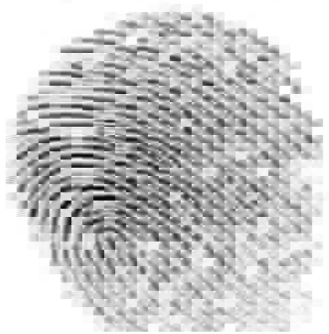

Figure 3.7:Fingerprint Enhanced by DCT 


\section{RESULTS}

Performance analysis of Latent finger print matching using FFT \& DCT

Table 4.1 Finger Print Matching using FFT\&DCT

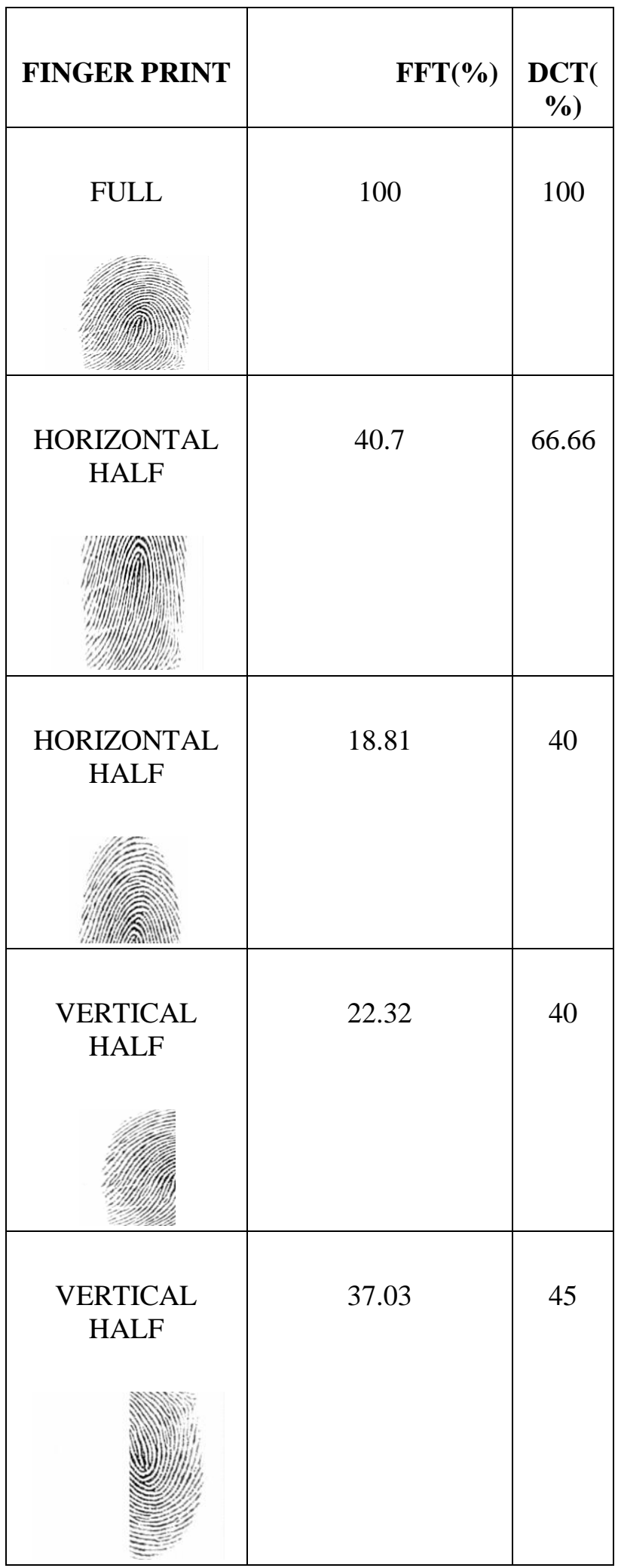

\begin{tabular}{|c|c|c|}
\hline FULL & 88.88 & 100 \\
\hline $\begin{array}{l}\text { HORIZONTAL } \\
\text { HALF }\end{array}$ & 9 & 33.33 \\
\hline $\begin{array}{c}\text { HORIZONTAL } \\
\text { HALF }\end{array}$ & 40 & 50 \\
\hline $\begin{array}{l}\text { VERTICAL } \\
\text { HALF }\end{array}$ & 28.43 & 40 \\
\hline $\begin{array}{l}\text { VERTICAL } \\
\text { HALF }\end{array}$ & 30 & 36 \\
\hline (u) & & \\
\hline
\end{tabular}

\section{CONCLUSION \& FUTURE SCOPE}

A new finger print matching technique planned for matching technique planned for matching latent towards plain/rolled finger prints using DCT \& FFT individually. The results shows that DCT gives better matching accuracy to FFT. The results can also be analyzed with other transforms such as DWT, DTCWT, SVD, and Iterative FFT individually or combination of two or more transforms, which are stated above.

\section{REFERENCES}

[1] P.Sony \& L.Padmalatha, "Latent Fingerprint Matching Based on Texture Descriptor using Hough Transform" in International Journal of computer Applications (09758887) Volume 106 No-17.NOV-2014. 
[2] A. A. Paulino, J. Feng \& A. K. Jain, "Latent fingerprint matching using descriptor-based Hough transform," in Proc. Int. Joint Conf. Biometrics, Oct. 2011.

[3] R. Cappelli, M. Ferrara, and D. Maltoni, "Minutiacylindercode:A new representation \& matching technique for fingerprint recognition," IEEE Trans.Pattern Anal. Mach. Intell., vol. 32, no. 12, pp.2128-2141, Dec. 2010.
[4] C. Champod, C. Lennard, P. Margot \& M. Stoilovic, Fingerprints and Other Ridge Skin Impressions. Boca Raton, FL: CRC Press, 2004.

[5] D. R. Ashbaugh, Quantitative-Qualitative Friction Ridge Analysis. Boca Raton, FL: CRC Press, 1999.

[6] X. Jiang and W.-Y. Yau, "Fingerprint minutiae matching based on the local \& global structures", in Proc. 15th Int. Conf. Pattern Recognition. 